AJMS

28,2

152

Received 13 January 2021

Revised 28 March 2021

Accepted 28 March 2021

\section{Arithmetic properties of singular overpartition pairs without multiples of $\boldsymbol{k}$}

\author{
S. Shivaprasada Nayaka, T.K. Sreelakshmi and Santosh Kumar \\ Department of Mathematics, BMS Institute of Technology and Management, \\ Bengaluru, India
}

Abstract

Purpose - In this paper, the author defines the function $\bar{B}_{i, j}^{\delta, k}(n)$, the number of singular overpartition pairs of $n$ without multiples of $k$ in which no part is divisible by $\delta$ and only parts congruent to $\pm i, \pm j$ modulo $\delta$ may be overlined.

Design/methodology/approach - Andrews introduced to combinatorial objects, which he called singular overpartitions and proved that these singular overpartitions depend on two parameters $\delta$ and $i$ can be enumerated by the function $\bar{C}_{\delta, i}(n)$, which gives the number of overpartitions of $n$ in which no part divisible by $\delta$ and parts $\equiv \pm i(\operatorname{Mod} \delta)$ may be overlined.

Findings - Using classical spirit of $q$-series techniques, the author obtains congruences modulo 4 for $\bar{B}_{2,4}^{8,3}(n)$, $\bar{B}_{2,4}^{8,5}$ and $\bar{B}_{2,4}^{12,3}$.

Originality/value - The results established in this work are extension to those proved in Andrews' singular overpatition pairs of $n$.

Keywords Congruences, Dissections, Singular overpartition pairs without multiples of $k$

Paper type Research paper

\section{Introduction}

For $|a b|<1$, Ramanujan's general theta function $f(a, b)$ is defined as

$$
f(a, b):=\sum_{n=-\infty}^{\infty} a^{n(n+1) / 2} b^{n(n-1) / 2},
$$

where the product representations arise from Jacobi's triple product identity [1, p. 35, Entry 19].

$$
f(a, b)=(-a ; a b)_{\infty}(-b ; a b)_{\infty}(a b ; a b)_{\infty} .
$$

Throughout the paper, we use the standard $q$-series notation, and $f_{k}$ is defined as

$$
f_{k}:=\left(q^{k} ; q^{k}\right)_{\infty}=\lim _{n \rightarrow \infty} \prod_{m=1}^{n}\left(1-q^{m k}\right) .
$$

The special cases of $f(a, b)$ are

\section{JEL Classification — 05A15, 05A17, 11P83}

(C) S. Shivaprasada Nayaka, T.K. Sreelakshmi and Santosh Kumar. Published in Arab Journal of Mathematical Sciences. Published by Emerald Publishing Limited. This article is published under the Creative Commons Attribution (CC BY 4.0) licence. Anyone may reproduce, distribute, translate and create derivative works of this article (for both commercial and non-commercial purposes), subject to full attribution to the original publication and authors. The full terms of this licence may be seen at http:// creativecommons.org/licences/by/4.0/legalcode

Dedicated to Prof. M. S. Mahadeva Naika on his 62 nd birthday.
Arab Journal of Mathematical Sciences

Vol. 28 No. 2, 2022 pp. $152-167$

Emerald Publishing Limited e-ISSN: 2588-9214

p-ISSN: 1319-5166

DOI 10.1108/AJMS-01-2021-0013 


$$
\begin{gathered}
\varphi(q):=f(q, q)=\sum_{n=-\infty}^{\infty} q^{n^{2}}=\left(-q ; q^{2}\right)_{\infty}^{2}\left(q^{2} ; q^{2}\right)_{\infty}=\frac{f_{2}^{5}}{f_{1}^{2} f_{4}^{2}} \\
\psi(q):=f\left(q, q^{3}\right)=\sum_{n=0}^{\infty} q^{n(n+1) / 2}=\frac{\left(q^{2} ; q^{2}\right)_{\infty}}{\left(q ; q^{2}\right)_{\infty}}=\frac{f_{2}^{2}}{f_{1}}
\end{gathered}
$$

and

$$
f(-q):=f\left(-q,-q^{2}\right)=\sum_{n=-\infty}^{\infty}(-1)^{n} q^{n(3 n-1) / 2}=(q ; q)_{\infty}=f_{1} .
$$

A partition of a positive integer $n$ is a nonincreasing sequence of positive integers whose sum is $n$. An overpartition, introduced by Corteel and Lovejoy [2], of a nonnegative integer $n$ is a nonincreasing sequence of natural numbers whose sum is $n$ in which the first occurrence of a number may be overlined.

Recently, G. E. Andrews [3] defined combinatorial objects, which he called singular overpartitions and proved that these singular overpartitions depend on two parameters $\delta$ and $i$ can be enumerated by the function $\bar{C}_{\delta, i}(n)$, which gives the number of overpartitions of $n$ in which no part divisible by $\delta$ and parts $\equiv \pm i(\bmod \delta)$ may be overlined. The generating function of $\bar{C}_{\delta, i}(n)$ is

$$
\sum_{n=0}^{\infty} \bar{C}_{\delta, i}(n) q^{n}=\frac{\left(q^{\delta} ; q^{\delta}\right)_{\infty}\left(-q^{i} ; q^{\delta}\right)_{\infty}\left(-q^{\delta-i} ; q^{\delta}\right)_{\infty}}{(q ; q)_{\infty}} .
$$

He also proved that

$$
\bar{C}_{3,1}(9 n+3) \equiv \bar{C}_{3,1}(9 n+6) \equiv 0(\bmod 3) .
$$

Andrews [3] proves that, for all $n \geq 0, \bar{C}_{3,1}(n)=\bar{A}_{3}(n)$, where $\bar{A}_{3}(n)$ is the number of overpartitions of $n$ into parts not divisible by 3 . The function $\bar{A}_{\ell}(n)$, which counts the number of overpartitions of $n$ into parts not divisible by $\ell$, plays a key role in the work of Lovejoy [4].

Chen et al. [5] have generalized (1.7) and proved some congruences modulo 2, 3, 4 and 8 for $\bar{C}_{3,1}(n)$. They also proved some congruence for $\bar{C}_{4,1}(n), \bar{C}_{6,1}(n)$ and $\bar{C}_{6,2}(n)$ modulo powers of 2 and 3. More recently, Ahmed and Baruah [6] have found some new congruences for $\bar{C}_{3,1}(n)$, $\bar{C}_{8,2}(n), \bar{C}_{12,4}(n), \bar{C}_{24,8}(n)$ and $\bar{C}_{48,16}(n)$ modulo 18,36 . Chen [7] has also found some congruences modulo powers of 2 for $\bar{C}_{3,1}(n), \bar{C}_{4,1}(n)$. Yao [8] has proved congruences modulo 16, 32, 64 for $\bar{C}_{3,1}(n)$. Naika and Gireesh [9] have found some congruences modulo 6, 12, 16, 18, 24,48 and 72 for $\bar{C}_{3,1}(n)$. Naika and Nayaka [10] have proved some congruences for $\overline{C O}_{3,1}(n)$ modulo powers of 2 and 3 . They have also proved in a paper [11] modulo 4 for $\bar{C}_{4,1}^{3}(n)$ and $\bar{C}_{4,1}^{5}(n)$.

In $[12,13]$, Naika et al. have defined the Andrews' singular overpartition pairs of $n$. Let $\bar{C}_{i, j}^{\delta}(n)$ denote the number of Andrews' singular overpartition pairs of $n$ in which no part is divisible by $\delta$ and only parts congruent to $\pm i, \pm j$ modulo $\delta$ may be overlined. Andrews' singular overpartition pair $\pi$ of $n$ is a pair of Andrews' singular overpartitions $(\lambda, \mu)$ such that the sum of all of the parts is $n$. They also established Ramanujan-like congruences for $\bar{A}_{1,2}^{6}(n)$ modulo 3,9, 27 and infinite families of congruences for $\bar{A}_{1,5}^{12}(n)$ modulo 4, 6 and 9 . 
AJMS 28,2
In this paper, we define the function $\bar{B}_{i, j}^{\delta, k}(n)$, the number of singular overpartition pairs of $n$ without multiples of $k$ in which no part is divisible by $\delta$ and only parts congruent to $\pm i, \pm j$ modulo $\delta$ may be overlined. The generating function of $\bar{B}_{i, j}^{\delta, k}(n)$ is given by

$$
\sum_{n=0}^{\infty} \bar{B}_{i, j}^{\delta, k}(n) q^{n}=\frac{f\left(q^{i}, q^{\delta-i}\right) f\left(q^{j}, q^{\delta-j}\right)\left(q^{k} ; q^{k}\right)_{\infty}^{2}}{f\left(q^{k i}, q^{k(\delta-i)}\right) f\left(q^{k j}, q^{k(\delta-j)}\right)(q ; q)_{\infty}^{2}} .
$$

In this paper, we establish some congruences modulo 4 for $\bar{B}_{2,4}^{8,3}(n), \bar{B}_{2,4}^{8,5}$ and $\bar{B}_{2,4}^{12,3}$. The main results of this paper can be stated as follows.

Theorem 1.1. For all integers $\alpha \geq 0$ and $n \geq 0$,

$$
\begin{aligned}
& \bar{B}_{2,4}^{8,3}(16 n+9) \equiv 0(\bmod 4), \\
& \bar{B}_{2,4}^{8,3}(16 n+13) \equiv 0(\bmod 4), \\
& \bar{B}_{2,4}^{8,3}(32 n+23) \equiv 0(\bmod 4), \\
& \bar{B}_{2,4}^{8,3}(32 n+31) \equiv 0(\bmod 4), \\
& \bar{B}_{2,4}^{8,3}(64 n+35) \equiv 0(\bmod 4), \\
& \bar{B}_{2,4}^{8,3}(64 n+51) \equiv 0(\bmod 4), \\
& \bar{B}_{2,4}^{8,3}(256 n+139) \equiv 0(\bmod 4), \\
& \bar{B}_{2,4}^{8,3}(256 n+203) \equiv 0(\bmod 4), \\
& \bar{B}_{2,4}^{8,3}(4 n+3) \equiv \bar{B}_{2,4}^{8,3}(64 n+43)(\bmod 4), \\
& \bar{B}_{2,4}^{8,3}(8 n+5) \equiv \bar{B}_{2,4}^{8,3}(32 n+19) \equiv \bar{B}_{2,4}^{8,3}(128+75)(\bmod 4) .
\end{aligned}
$$

Theorem 1.2. Let $p$ be a prime $\geq 5,\left(\frac{-6}{p}\right)=-1$. Then for all integers $\alpha \geq 1$, and $n \geq 0$,

$$
\sum_{n=0}^{\infty} \bar{B}_{2,4}^{8,3}\left(16 p^{2 \alpha} n+\frac{14 p^{2 \alpha}+1}{3}\right) q^{n} \equiv 2 f_{1} f_{6}(\bmod 4) .
$$

Theorem 1.3. Let $p$ be a prime $\geq 5,\left(\frac{-6}{p}\right)=-1$. Then for all integers $\alpha \geq 0$, and $n \geq 0$,

$$
\bar{B}_{2,4}^{8,3}\left(16 p^{2 \alpha+2} n+16 p^{2 \alpha+1} i+\frac{14 p^{2 \alpha+2}+1}{3}\right) \equiv 0(\bmod 4)
$$

where $i$ is an integer and $1 \leq i \leq p-1$.

Theorem 1.4. For all integers $\alpha \geq 0$ and $n \geq 0$,

$$
\bar{B}_{2,4}^{8,5}(4 n+3) \equiv 0(\bmod 4),
$$




$$
\bar{B}_{2,4}^{8,5}(8 n+5) \equiv 0(\bmod 4) .
$$

Theorem 1.5. For any prime $p \geq 5, \alpha \geq 0$ and $n \geq 0$, we have

$$
\sum_{n=0}^{\infty} \bar{B}_{2,4}^{8,5}\left(8 p^{2 \alpha} n+\frac{p^{3 \alpha}+2}{3}\right) q^{n} \equiv 2 f_{1}(\bmod 4) .
$$

Theorem 1.6. For any prime $p \geq 5, \alpha \geq 0, n \geq 0$ and $l=1,2, \ldots p-1$, we have

Singular overpartition pairs without multiples of $k$

$$
\bar{B}_{2,4}^{8,5}\left(8 p^{2 \alpha+1}(p n+l)+\frac{p^{3 \alpha}+2}{3}\right) \equiv 0(\bmod 4) \text {. }
$$

Theorem 1.7. For all integers $\alpha \geq 0$ and $n \geq 0$,

$$
\begin{gathered}
\bar{B}_{2,4}^{12,3}(6 n+5) \equiv 0(\bmod 4), \\
\bar{B}_{2,4}^{12,3}(12 n+9) \equiv 0(\bmod 4), \\
\bar{B}_{2,4}^{12,3}(36 n+27) \equiv 0(\bmod 4), \\
\bar{B}_{2,4}^{12,3}(108 n+51) \equiv 0(\bmod 4), \\
\bar{B}_{2,4}^{12,3}(108 n+87) \equiv 0(\bmod 4), \\
\bar{B}_{2,4}^{12,3}\left(4 \cdot 3^{2 \alpha+3} n+\frac{32 \cdot 3^{2 \alpha+3}+3}{2}\right) \equiv 0(\bmod 4) .
\end{gathered}
$$

Theorem 1.8. For any prime $p \geq 5, \alpha \geq 0$ and $n \geq 0$, we have

$$
\sum_{n=0}^{\infty} \bar{B}_{2,4}^{12,3}\left(36 p^{2 \alpha} n+\frac{3\left(p^{3 \alpha}+1\right)}{2}\right) q^{n} \equiv 2 f_{1}(\bmod 4) .
$$

Theorem 1.9. For any prime $p \geq 5, \alpha \geq 0, n \geq 0$ and $l=1,2, \ldots p-1$, we have

$$
\bar{B}_{2,4}^{12,3}\left(36 p^{2 \alpha+1}(p n+l)+\frac{3\left(p^{3 \alpha}+1\right)}{2}\right) \equiv 0(\bmod 4) \text {. }
$$

\section{Preliminary results}

We need the following few dissection formulas to prove our main results,

Lemma 2.1. The following two dissections hold:

$$
\begin{gathered}
\frac{f_{3}^{3}}{f_{1}}=\frac{f_{4}^{3} f_{6}^{2}}{f_{2}^{2} f_{12}}+q \frac{f_{12}^{3}}{f_{4}}, \\
\frac{f_{3}}{f_{1}^{3}}=\frac{f_{4}^{6} f_{6}^{3}}{f_{2}^{9} f_{12}^{2}}+3 q \frac{f_{4}^{2} f_{6} f_{12}^{2}}{f_{2}^{7}} .
\end{gathered}
$$


Lemma 2.2. The following two dissections hold:

$$
\frac{1}{f_{1} f_{3}}=\frac{f_{8}^{2} f_{12}^{5}}{f_{2}^{2} f_{4} f_{6}^{4} f_{24}^{2}}+q \frac{f_{4}^{5} f_{24}^{2}}{f_{2}^{4} f_{6}^{2} f_{8}^{2} f_{12}},
$$

$$
f_{1} f_{3}=\frac{f_{2} f_{8}^{2} f_{12}^{4}}{f_{4}^{2} f_{6} f_{24}^{2}}-q \frac{f_{4}^{4} f_{6} f_{24}^{2}}{f_{2} f_{8}^{2} f_{12}^{2}} .
$$

Eqn (2.3) was proved by Baruah and Ojah [16]. Replacing $q$ by $-q$ in (2.3) and using the fact that $(-q ;-q)_{\infty}=\frac{f_{2}^{3}}{f_{1} f_{4}}$, we get (2.4).

Lemma 2.3. The following two dissections hold:

$$
\begin{gathered}
\frac{f_{3}}{f_{1}}=\frac{f_{4} f_{6} f_{16} f_{24}^{2}}{f_{2}^{2} f_{8} f_{12} f_{48}}+q \frac{f_{6} f_{8}^{2} f_{48}}{f_{2}^{2} f_{16} f_{24}}, \\
\frac{f_{1}}{f_{3}}=\frac{f_{2} f_{16} f_{24}^{2}}{f_{6}^{2} f_{8} f_{48}}-q \frac{f_{2} f_{8}^{2} f_{12} f_{48}}{f_{4} f_{6}^{2} f_{16} f_{24}} .
\end{gathered}
$$

Xia and Yao [17] gave a proof of Lemma (2.3). Replacing $q$ by $-q$ in (2.5) and using the fact that $(-q ;-q)_{\infty}=\frac{f_{2}^{3}}{f_{1} f_{4}}$, we get (2.6).

Lemma 2.4. The following two dissections hold:

$$
\begin{gathered}
\frac{f_{3}^{2}}{f_{1}^{2}}=\frac{f_{4}^{4} f_{6} f_{12}^{2}}{f_{2}^{5} f_{8} f_{24}}+2 q \frac{f_{4} f_{6}^{2} f_{8} f_{24}}{f_{2}^{4} f_{12}}, \\
\frac{f_{1}^{2}}{f_{3}^{2}}=\frac{f_{2} f_{4}^{2} f_{12}^{4}}{f_{6}^{5} f_{8} f_{24}}-2 q \frac{f_{2}^{2} f_{8} f_{12} f_{24}}{f_{4} f_{6}^{4}} .
\end{gathered}
$$

Xia and Yao [18] proved (2.7) by employing an addition formula for theta functions.

Lemma 2.5. The following two dissections hold:

$$
\frac{f_{5}}{f_{1}}=\frac{f_{8} f_{20}^{2}}{f_{2}^{2} f_{40}}+q \frac{f_{4}^{3} f_{10} f_{40}}{f_{2}^{3} f_{8} f_{20}} .
$$

Eqn (2.9) was proved by Hirschhorn and Sellers [19].

Lemma 2.6. The following three dissections hold:

$$
f_{1} f_{2}=\frac{f_{6} f_{9}^{4}}{f_{3} f_{18}^{2}}-q f_{9} f_{18}-2 q^{2} \frac{f_{3} f_{18}^{4}}{f_{6} f_{9}^{2}} .
$$

One can see this identity in [20]. 
Lemma 2.7. (Cui and $\mathrm{Gu}[21$, Theorem 2.2]). For any prime $p \geq 5$,

$$
f_{1}=\sum_{\substack{k=\frac{1-p}{2} \\ k \neq \frac{p-1}{6}}}^{\frac{p-1}{2}}(-1)^{k} q^{\frac{3 k^{2}+k}{2}} f\left(-q^{\frac{3 p^{2}+(6 k+1) p}{2}},-q^{\frac{3 p^{2}-(6 k+1) p}{2}}\right)+(-1)^{\frac{ \pm p-1}{6}} q^{\frac{p^{2}-1}{24}} f_{p^{2}}
$$

Singular overpartition pairs without multiples of $k$

where

$$
\frac{ \pm p-1}{6}:= \begin{cases}\frac{p-1}{6}, & \text { if } p \equiv 1(\bmod 6) \\ \frac{-p-1}{6}, & \text { if } p \equiv-1(\bmod 6)\end{cases}
$$

Lemma 2.8. For any prime $p$ and positive integer $n$,

$$
f_{1}^{p^{n}} \equiv f_{p}^{p^{n-1}}\left(\bmod p^{n}\right)
$$

\section{Proof of Theorem (1.1)}

Setting $i=2, j=4, \delta=8$ and $k=3$ in (1.8), we see that

$$
\sum_{n=0}^{\infty} \bar{B}_{2,4}^{8,3}(n) q^{n}=\frac{f\left(q^{2}, q^{6}\right) f\left(q^{4}, q^{4}\right)\left(q^{3} ; q^{3}\right)_{\infty}^{2}}{f\left(q^{6}, q^{18}\right) f\left(q^{12}, q^{12}\right)(q ; q)_{\infty}^{2}} .
$$

By the definition of $f(a, b)$ and the well-known Jacobi triple product identity, we get

$$
\sum_{n=0}^{\infty} \bar{B}_{2,4}^{8,3}(n) q^{n}=\frac{f_{3}^{2} f_{6} f_{8}^{5} f_{48}^{2}}{f_{1}^{2} f_{2} f_{16}^{2} f_{24}^{5}}
$$

Substituting (2.7) into (3.2), we have

$$
\sum_{n=0}^{\infty} \bar{B}_{2,4}^{8,3}(n) q^{n}=\frac{f_{6}^{2} f_{8}^{4} f_{48}^{2} f_{4}^{4} f_{12}^{2}}{f_{2}^{6} f_{16}^{2} f_{24}^{6}}+2 q \frac{f_{6}^{3} f_{8}^{6} f_{4} f_{48}^{2}}{f_{2}^{5} f_{16}^{2} f_{24}^{4} f_{12}} .
$$

Equating odd parts of the aforementioned equation, we obtain

$$
\sum_{n=0}^{\infty} \bar{B}_{2,4}^{8,3}(2 n+1) q^{n}=2 \frac{f_{3}^{3} f_{4}^{6} f_{2} f_{24}^{2}}{f_{1}^{5} f_{8}^{2} f_{12}^{4} f_{6}} .
$$

Involving (2.11) in (3.4), we get

$$
\sum_{n=0}^{\infty} \bar{B}_{2,4}^{8,3}(2 n+1) q^{n} \equiv 2 \frac{f_{3} f_{4}^{2}}{f_{1}^{3}}(\bmod 4) .
$$

Employing (2.2) into (3.5), we arrive at

$$
\sum_{n=0}^{\infty} \bar{B}_{2,4}^{8,3}(2 n+1) q^{n} \equiv 2 \frac{f_{4}^{8} f_{6}^{3}}{f_{2}^{9} f_{12}^{2}}+2 q \frac{f_{4}^{4} f_{6} f_{12}}{f_{2}^{7}}(\bmod 4) .
$$


AJMS

28,2
Extracting the terms involving $q^{2 n}$ from both sides of (3.6), we have

$$
\sum_{n=0}^{\infty} \bar{B}_{2,4}^{8,3}(4 n+1) q^{n} \equiv 2 \frac{f_{2}^{8} f_{3}^{3}}{f_{1}^{9} f_{6}^{2}}(\bmod 4) .
$$

Using (2.11) in the aforementioned equation, we obtain

Substituting (2.1) into (3.8), we find that

$$
\sum_{n=0}^{\infty} \bar{B}_{2,4}^{8,3}(4 n+1) q^{n} \equiv 2 \frac{f_{2}^{2} f_{4}^{3}}{f_{12}}+2 q \frac{f_{2}^{4} f_{12}^{3}}{f_{4} f_{6}^{2}}(\bmod 4),
$$

which implies

$$
\sum_{n=0}^{\infty} \bar{B}_{2,4}^{8,3}(8 n+1) q^{n} \equiv 2 \frac{f_{1}^{2} f_{2}^{3}}{f_{6}}(\bmod 4) .
$$

Invoking (2.11) in (3.10), the equation reduces to

$$
\sum_{n=0}^{\infty} \bar{B}_{2,4}^{8,3}(8 n+1) q^{n} \equiv 2 \frac{f_{1}^{2} f_{2}^{3}}{f_{3}^{2}}(\bmod 4) .
$$

Employing (2.8) into (3.11), we obtain

$$
\sum_{n=0}^{\infty} \bar{B}_{2,4}^{8,3}(8 n+1) q^{n} \equiv 2 \frac{f_{2}^{4} f_{4}^{2} f_{12}^{4}}{f_{6}^{5} f_{8} f_{24}}(\bmod 4) .
$$

Congruence (1.9) easily follows from the aforementioned equation.

From (3.6), we have

$$
\sum_{n=0}^{\infty} \bar{B}_{2,4}^{8,3}(4 n+3) q^{n} \equiv 2 \frac{f_{2}^{4} f_{3} f_{6}^{2}}{f_{1}^{7}}(\bmod 4) .
$$

Using (2.11) in (3.6), we found

$$
\sum_{n=0}^{\infty} \bar{B}_{2,4}^{8,3}(4 n+3) q^{n} \equiv 2 \frac{f_{3} f_{2} f_{12}}{f_{1}}(\bmod 4) .
$$

Substituting (2.5) into (3.14), we obtain

$$
\sum_{n=0}^{\infty} \bar{B}_{2,4}^{8,3}(4 n+3) q^{n} \equiv 2 \frac{f_{6}^{3} f_{4} f_{16} f_{24}^{2}}{f_{2} f_{8} f_{12} f_{48}}+2 q \frac{f_{6}^{3} f_{8}^{2} f_{48}}{f_{2} f_{16} f_{24}}(\bmod 4) .
$$

Extracting the terms involving $q^{2 n+1}$ from (3.15), dividing by $q$ and replacing $q^{2}$ by $q$, we arrive at

$$
\sum_{n=0}^{\infty} \bar{B}_{2,4}^{8,3}(8 n+7) q^{n} \equiv 2 \frac{f_{3}^{3} f_{4}^{2} f_{24}}{f_{1} f_{8} f_{12}}(\bmod 4) .
$$


Invoking (2.11) in (3.16), we get

$$
\sum_{n=0}^{\infty} \bar{B}_{2,4}^{8,3}(8 n+7) q^{n} \equiv 2 \frac{f_{3}^{3} f_{12}}{f_{1}}(\bmod 4) .
$$
overpartition pairs without multiples of $k$

Employing (2.1) into (3.17), we obtain

$$
\sum_{n=0}^{\infty} \bar{B}_{2,4}^{8,3}(8 n+7) q^{n} \equiv 2 \frac{f_{4}^{3} f_{6}^{2}}{f_{2}^{2}}+2 q \frac{f_{12}^{4}}{f_{4}}(\bmod 4) .
$$

Extracting the terms involving $q^{2 n}$ from both sides of (3.18), we have

$$
\sum_{n=0}^{\infty} \bar{B}_{2,4}^{8,3}(16 n+7) q^{n} \equiv 2 \frac{f_{2}^{3} f_{3}^{2}}{f_{1}^{2}}(\bmod 4) .
$$

Using (2.11) in (3.19), we arrive at

$$
\sum_{n=0}^{\infty} \bar{B}_{2,4}^{8,3}(16 n+7) q^{n} \equiv 2 f_{2} f_{6}(\bmod 4) .
$$

Congruence (1.11) easily follows from (3.20).

From (3.18), we have

$$
\sum_{n=0}^{\infty} \bar{B}_{2,4}^{8,3}(16 n+15) q^{n} \equiv 2 \frac{f_{6}^{4}}{f_{2}}(\bmod 4) .
$$

Congruence (1.12) follows by extracting the terms involving $q^{2 n+1}$ from (3.21).

From (3.15), we get

$$
\sum_{n=0}^{\infty} \bar{B}_{2,4}^{8,3}(8 n+3) q^{n} \equiv 2 \frac{f_{3}^{3} f_{2} f_{8} f_{12}^{2}}{f_{1} f_{4} f_{6} f_{24}}(\bmod 4) .
$$

Using (2.11) in (3.22), we obtain

$$
\sum_{n=0}^{\infty} \bar{B}_{2,4}^{8,3}(8 n+3) q^{n} \equiv 2 \frac{f_{3}^{3} f_{2} f_{4}}{f_{1} f_{6}}(\bmod 4) .
$$

Employing (2.1) into (3.23), we reduce that

$$
\sum_{n=0}^{\infty} \bar{B}_{2,4}^{8,3}(8 n+3) q^{n} \equiv 2 \frac{f_{4}^{4} f_{6}}{f_{2} f_{12}}+2 q \frac{f_{2} f_{12}^{3}}{f_{6}}(\bmod 4) .
$$

Extracting the terms involving $q^{2 n}$ from both sides of (3.24), we find that

$$
\sum_{n=0}^{\infty} \bar{B}_{2,4}^{8,3}(16 n+3) q^{n} \equiv 2 \frac{f_{3} f_{2}^{4}}{f_{1} f_{6}}(\bmod 4) .
$$

Substituting (2.5) into (3.25), we arrive at

$$
\sum_{n=0}^{\infty} \bar{B}_{2,4}^{8,3}(16 n+3) q^{n} \equiv 2 \frac{f_{2}^{2} f_{4} f_{16} f_{24}^{2}}{f_{8} f_{12} f_{48}}+2 q \frac{f_{2}^{2} f_{8}^{2} f_{48}}{f_{16} f_{24}}(\bmod 4),
$$


which implies

28,2

$$
\sum_{n=0}^{\infty} \bar{B}_{2,4}^{8,3}(32 n+3) q^{n} \equiv 2 \frac{f_{1}^{2} f_{2} f_{8} f_{12}^{2}}{f_{4} f_{6} f_{24}}(\bmod 4)
$$

Invoking (2.11) in (3.27), we obtain

$$
\sum_{n=0}^{\infty} \bar{B}_{2,4}^{8,3}(32 n+3) q^{n} \equiv 2 \frac{f_{8}}{f_{6}}(\bmod 4) .
$$

Congruence (1.13) follows by extracting the terms involving $q^{2 n+1}$ from (3.28).

Extracting the terms involving $q^{2 n+1}$ from (3.26), dividing by $q$ and replacing $q^{2}$ by $q$, we arrive at

$$
\sum_{n=0}^{\infty} \bar{B}_{2,4}^{8,3}(32 n+19) q^{n} \equiv 2 \frac{f_{1}^{2} f_{4}^{2} f_{24}}{f_{8} f_{12}}(\bmod 4) .
$$

Using (2.11) in (3.29), we get

$$
\sum_{n=0}^{\infty} \bar{B}_{2,4}^{8,3}(32 n+19) q^{n} \equiv 2 f_{2} f_{12}(\bmod 4) .
$$

Congruence (1.14) follows from (3.30).

Extracting the terms involving $q^{2 n}$ from both sides of (3.24), we have

$$
\sum_{n=0}^{\infty} \bar{B}_{2,4}^{8,3}(16 n+11) q^{n} \equiv 2 \frac{f_{1} f_{6}^{3}}{f_{3}}(\bmod 4) .
$$

Employing (2.6) into (3.31), we found

$$
\sum_{n=0}^{\infty} \bar{B}_{2,4}^{8,3}(16 n+11) q^{n} \equiv 2 \frac{f_{2} f_{6} f_{16} f_{24}^{2}}{f_{8} f_{48}}+2 q \frac{f_{2} f_{6} f_{8}^{2} f_{12} f_{48}}{f_{4} f_{16} f_{24}}(\bmod 4)
$$

which implies

$$
\sum_{n=0}^{\infty} \bar{B}_{2,4}^{8,3}(32 n+11) q^{n} \equiv 2 \frac{f_{1} f_{3} f_{8} f_{12}^{2}}{f_{4} f_{24}}(\bmod 4) .
$$

Substituting (2.4) into (3.33), we get

$$
\sum_{n=0}^{\infty} \bar{B}_{2,4}^{8,3}(32 n+11) q^{n} \equiv 2 \frac{f_{2} f_{8}^{3} f_{12}^{6}}{f_{4}^{3} f_{6} f_{24}^{3}}+2 q \frac{f_{4}^{3} f_{6} f_{24}}{f_{2} f_{8}}(\bmod 4),
$$

which implies

$$
\sum_{n=0}^{\infty} \bar{B}_{2,4}^{8,3}(64 n+11) q^{n} \equiv 2 \frac{f_{1} f_{4}^{3} f_{6}^{6}}{f_{2}^{3} f_{3} f_{12}^{3}}(\bmod 4) .
$$

Using (2.11) in (3.35), we find that

$$
\sum_{n=0}^{\infty} \bar{B}_{2,4}^{8,3}(64 n+11) q^{n} \equiv 2 \frac{f_{1}}{f_{3} f_{2}^{3}}(\bmod 4) .
$$


Employing (2.6) into (3.36), we get

$$
\sum_{n=0}^{\infty} \bar{B}_{2,4}^{8,3}(64 n+11) q^{n} \equiv 2 \frac{f_{2}^{4} f_{16} f_{24}^{2}}{f_{6}^{2} f_{8} f_{48}}+2 q \frac{f_{2}^{4} f_{8}^{2} f_{12} f_{48}}{f_{4} f_{6}^{2} f_{16} f_{24}}(\bmod 4) .
$$

Extracting the terms involving $q^{2 n}$ from (3.37) and replacing $q^{2}$ by $q$, we obtain

$$
\sum_{n=0}^{\infty} \bar{B}_{2,4}^{8,3}(128 n+11) q^{n} \equiv 2 \frac{f_{1}^{4} f_{8} f_{12}^{2}}{f_{3}^{2} f_{4} f_{24}}(\bmod 4) .
$$

Invoking (2.11) in (3.38), we arrive at

$$
\sum_{n=0}^{\infty} \bar{B}_{2,4}^{8,3}(128 n+11) q^{n} \equiv 2 \frac{f_{2}^{2} f_{8}}{f_{4} f_{6}}(\bmod 4) .
$$

Congruence (1.15) follows by extracting the terms involving $q^{2 n+1}$ from (3.39).

From (3.37), we have

$$
\sum_{n=0}^{\infty} \bar{B}_{2,4}^{8,3}(128 n+75) q^{n} \equiv 2 \frac{f_{1}^{4} f_{4}^{2} f_{6} f_{24}}{f_{2} f_{3}^{2} f_{8} f_{12}}(\bmod 4) .
$$

Invoking (2.11) in (3.40), we obtain

$$
\sum_{n=0}^{\infty} \bar{B}_{2,4}^{8,3}(128 n+75) q^{n} \equiv 2 f_{2} f_{12}(\bmod 4) .
$$

Congruence (1.16) follows from (3.41).

From (3.34), we arrive at

$$
\sum_{n=0}^{\infty} \bar{B}_{2,4}^{8,3}(64 n+43) q^{n} \equiv 2 \frac{f_{2}^{3} f_{3} f_{12}}{f_{1} f_{4}}(\bmod 4)
$$

Using (2.11) in (3.42), we get

$$
\sum_{n=0}^{\infty} \bar{B}_{2,4}^{8,3}(64 n+43) q^{n} \equiv 2 \frac{f_{3} f_{2} f_{12}}{f_{1}}(\bmod 4) .
$$

From the equations (3.14) and (3.43), we obtain (1.17).

Extracting the terms involving $q^{2 n+1}$ from (3.9), dividing by $q$ and replacing $q^{2}$ by $q$, we obtain

$$
\sum_{n=0}^{\infty} \bar{B}_{2,4}^{8,3}(8 n+5) q^{n} \equiv 2 \frac{f_{1}^{4} f_{6}^{3}}{f_{2} f_{3}^{2}}(\bmod 4)
$$

Invoking (2.11) in (3.44), we get

$$
\sum_{n=0}^{\infty} \bar{B}_{2,4}^{8,3}(8 n+5) q^{n} \equiv 2 f_{2} f_{12}(\bmod 4) .
$$

Congruence (1.10) follows by extracting the terms involving $q^{2 n+1}$ from (3.45).

From equations (3.45), (3.30) and (3.40), we obtain (1.18). 
AJMS

28,2

162

4. Proof of Theorem (1.2)

Extracting the terms involving $q^{2 n}$ from (3.45) and replacing $q^{2}$ by $q$, we have

$$
\sum_{n=0}^{\infty} \bar{B}_{2,4}^{8,3}(16 n+5) q^{n} \equiv 2 f_{1} f_{6}(\bmod 4) .
$$

define

$$
\sum_{n=0}^{\infty} g(n) q^{n}=f_{1} f_{6}
$$

Combining (4.1) and (4.2), we find that

$$
\sum_{n=0}^{\infty} \bar{B}_{2,4}^{8,3}(16 n+5) q^{n} \equiv 2 \sum_{n=0}^{\infty} g(n) q^{n}(\bmod 4) .
$$

For a prime, $p \geq 5$ or $\frac{-(p-1)}{2} \leq k, m \leq \frac{p-1}{2}$, consider

$$
\frac{3 k^{2}+k}{2}+6 \cdot \frac{3 m^{2}+m}{2} \equiv \frac{7 p^{2}-7}{24}(\bmod p),
$$

therefore,

$$
(6 k+1)^{2}+6 \cdot(6 m+1)^{2} \equiv 0(\bmod p),
$$

Since $\left(\frac{-6}{p}\right)=-1$ the congruence relation (4.4) holds if and only if both $k=m=\frac{ \pm p-1}{6}$. Therefore, if we substitute Lemma (2.7) into (4.2) and then extract the terms in which the powers of $q$ are congruent to $\frac{7 p^{2}-7}{24}$ modulo $p$ and then divide by $q^{\frac{7 p^{2}-7}{24}}$, we find that

$$
\sum_{n=0}^{\infty} g\left(p n+\frac{7 p^{2}-7}{24}\right) q^{p n}=f_{p^{2}} f_{6 p^{2}}
$$

which implies that

$$
\sum_{n=0}^{\infty} g\left(p^{2} n+\frac{7 p^{2}-7}{24}\right) q^{n}=f_{1} f_{6}
$$

and for $n \geq 0$,

$$
g\left(p^{2} n+p i+\frac{7 p^{2}-7}{24}\right)=0,
$$

where $i$ is an integer and $1 \leq i \leq p-1$. By induction, we see that for $n \geq 0$ and $\alpha \geq 0$,

$$
g\left(p^{2 \alpha} n+\frac{7 p^{2 \alpha}-7}{24}\right)=g(n) .
$$

Replacing $n$ by $p^{2 \alpha} n+\frac{7 p^{2 \alpha}-7}{24}$ in (4.3), we arrive at (1.19). 
5. Proof of Theorem (1.3)

Replacing $n$ by $p^{2} n+p i+\frac{7 p^{2}-7}{24}$ in (4.7) and using (4.6), we find that for $n \geq 0$ and $\alpha \geq 0$,

$$
g\left(p^{2 \alpha+2} n+p^{2 \alpha+1} i+\frac{7 p^{\alpha+2}-7}{24}\right)=0 .
$$

Comparing coefficients of $q^{n}$ from both sides of (4.3), we see that for $n \geq 0$,

$$
\bar{B}_{2,4}^{8,3}(16 n+5) \equiv 2 g(n)(\bmod 4) .
$$

The required result follows from (5.1) and (5.2).

\section{Proof of Theorem (1.4)}

Setting $i=2, j=4, \delta=8$ and $k=5$ in (1.8), we see that

$$
\sum_{n=0}^{\infty} \bar{B}_{2,4}^{8,5}(n) q^{n}=\frac{f\left(q^{2}, q^{6}\right) f\left(q^{4}, q^{4}\right)\left(q^{5} ; q^{5}\right)_{\infty}^{2}}{f\left(q^{10}, q^{30}\right) f\left(q^{20}, q^{20}\right)(q ; q)_{\infty}^{2}} .
$$

By the definition of $f(a, b)$ and the well-known Jacobi triple product identity, we get

$$
\sum_{n=0}^{\infty} \bar{B}_{2,4}^{8,5}(n) q^{n}=\frac{f_{5}^{2} f_{8}^{5} f_{10} f_{80}^{2}}{f_{1}^{2} f_{2} f_{16}^{2} f_{40}^{5}}
$$

Substituting (2.9) into (6.2), we have

$$
\sum_{n=0}^{\infty} \bar{B}_{2,4}^{8,5}(n) q^{n}=\frac{f_{8}^{7} f_{10} f_{20}^{4} f_{80}^{2}}{f_{2}^{5} f_{16}^{2} f_{40}^{7}}+2 q \frac{f_{4}^{3} f_{8}^{5} f_{10}^{2} f_{20} f_{80}^{2}}{f_{2}^{6} f_{16}^{2} f_{40}^{5}}+q^{2} \frac{f_{4}^{6} f_{8}^{3} f_{10}^{3} f_{80}^{2}}{f_{2}^{7} f_{16}^{2} f_{40}^{3} f_{20}^{2}} .
$$

Equating odd parts of the aforementioned equation, we obtain

$$
\sum_{n=0}^{\infty} \bar{B}_{2,4}^{8,5}(2 n+1) q^{n}=2 \frac{f_{2}^{3} f_{4}^{5} f_{5}^{2} f_{10} f_{40}^{2}}{f_{1}^{6} f_{8}^{2} f_{20}^{5}} .
$$

Involving (2.11) in (6.4), we get

$$
\sum_{n=0}^{\infty} \bar{B}_{2,4}^{8,5}(2 n+1) q^{n} \equiv 2 f_{4}(\bmod 4)
$$

Congruences (1.21) and (1.22) follow from the aforementioned equation.

\section{Proof of Theorem (1.5)}

From (6.5), we have

$$
\sum_{n=0}^{\infty} \bar{B}_{2,4}^{8,5}(8 n+1) q^{n} \equiv 2 f_{1}(\bmod 4) .
$$

Employing Lemma (2.7) into (7.1), it can be see that

$$
\sum_{n=0}^{\infty} \bar{B}_{2,4}^{8,5}\left(8\left(p n+\frac{p^{2}-1}{24}\right)+1\right) q^{n} \equiv 2 f_{p}(\bmod 4),
$$


which implies

28,2

$$
\sum_{n=0}^{\infty} \bar{B}_{2,4}^{8,5}\left(8 p^{2} n+\frac{p^{3}+2}{3}\right) q^{n} \equiv 2 f_{1}(\bmod 4) .
$$

Therefore,

$$
\bar{B}_{2,4}^{8,5}\left(8 p^{2} n+\frac{p^{3}+2}{3}\right) \equiv \bar{B}_{2,4}^{8,5}(8 n+1)(\bmod 4)
$$

Using the aforementioned relation and by induction on $\alpha$, we arrive at (1.23).

\section{Proof of Theorem (1.6)}

Combining (7.2) with Theorem (1.5), we derive that for $\alpha \geq 0$,

$$
\sum_{n=0}^{\infty} \bar{B}_{2,4}^{8,5}\left(8 p^{2 \alpha+1} n+\frac{p^{3 \alpha}+2}{3}\right) \equiv 2 f_{p}(\bmod 4) .
$$

Therefore, it follows that

$$
\sum_{n=0}^{\infty} \bar{B}_{2,4}^{8,5}\left(8 p^{2 \alpha+1}(p n+l)+\frac{p^{3 \alpha}+2}{3}\right) \equiv 0(\bmod 4) .
$$

where $l=1,2, \ldots, p-1$, we obtain (1.24).

\section{Proof of Theorem (1.7)}

Setting $i=2, j=4, \delta=12$ and $k=3$ in (1.8), we see that

$$
\sum_{n=0}^{\infty} \bar{B}_{2,4}^{12,3}(n) q^{n}=\frac{f\left(q^{2}, q^{10}\right) f\left(q^{4}, q^{8}\right)\left(q^{3} ; q^{3}\right)_{\infty}^{2}}{f\left(q^{6}, q^{30}\right) f\left(q^{12}, q^{24}\right)(q ; q)_{\infty}^{2}} .
$$

By the definition of $f(a, b)$ and the well-known Jacobi triple product identity, we get

$$
\sum_{n=0}^{\infty} \bar{B}_{2,4}^{12,3}(n) q^{n}=\frac{f_{3}^{2} f_{4} f_{6}^{2}}{f_{1}^{2} f_{2} f_{18} f_{36}} .
$$

Substituting (2.7) into (9.2), we have

$$
\sum_{n=0}^{\infty} \bar{B}_{2,4}^{12,3}(n) q^{n}=\frac{f_{4}^{5} f_{6}^{3} f_{12}^{2}}{f_{2}^{6} f_{8} f_{18} f_{24} f_{36}}+2 q \frac{f_{4}^{2} f_{6}^{4} f_{8} f_{24}}{f_{2}^{5} f_{12} f_{18} f_{36}} .
$$

Equating odd parts of the aforementioned equation, we obtain

$$
\sum_{n=0}^{\infty} \bar{B}_{2,4}^{12,3}(2 n+1) q^{n}=2 \frac{f_{2}^{2} f_{3}^{4} f_{4} f_{12}}{f_{1}^{5} f_{6} f_{9} f_{18}} .
$$

Involving (2.11) in (9.4), we get

$$
\sum_{n=0}^{\infty} \bar{B}_{2,4}^{12,3}(2 n+1) q^{n} \equiv 2 \frac{f_{2}^{2} f_{6} f_{12}}{f_{1} f_{9} f_{18}}(\bmod 4) .
$$


Ramanujan recorded the following identity in his third note book:

$$
\psi(q)=\frac{f_{2}^{2}}{f_{1}}=\frac{f_{6} f_{9}^{2}}{f_{3} f_{18}}+q \frac{f_{18}^{2}}{f_{9}} .
$$

Substituting (9.6) into (9.5), we deduce that

$$
\sum_{n=0}^{\infty} \bar{B}_{2,4}^{12,3}(2 n+1) q^{n} \equiv 2 \frac{f_{6}^{2} f_{9} f_{12}}{f_{3} f_{18}^{2}}+2 q \frac{f_{6} f_{12} f_{18}}{f_{9}^{2}}(\bmod 4) .
$$

Congruence (1.25) easily follows from the aforementioned equation.

Extracting the terms involving $q^{3 n+1}$ from (9.7), dividing by $q$ and replacing $q^{3}$ by $q$, we arrive at

$$
\sum_{n=0}^{\infty} \bar{B}_{2,4}^{12,3}(6 n+3) q^{n} \equiv 2 \frac{f_{2} f_{4} f_{6}}{f_{3}^{2}}(\bmod 4)
$$

Using (2.11) in (9.8), we get

$$
\sum_{n=0}^{\infty} \bar{B}_{2,4}^{12,3}(6 n+3) q^{n} \equiv 2 f_{2} f_{4}(\bmod 4)
$$

Congruence (1.26) follows by extracting the terms involving $q^{2 n+1}$ from (9.9).

From (9.9), we can reduce that

$$
\sum_{n=0}^{\infty} \bar{B}_{2,4}^{12,3}(12 n+3) q^{n} \equiv 2 f_{1} f_{2}(\bmod 4) .
$$

Employing (2.10) into (9.10), we get

$$
\sum_{n=0}^{\infty} \bar{B}_{2,4}^{12,3}(12 n+3) q^{n} \equiv 2 \frac{f_{6} f_{9}^{4}}{f_{3} f_{18}^{2}}+2 q f_{9} f_{18}(\bmod 4) .
$$

Congruence (1.27) follows from (9.11). have

Extracting the terms involving $q^{3 n+1}$ from (9.11), dividing by $q$ and replacing $q^{3}$ by $q$, we

$$
\sum_{n=0}^{\infty} \bar{B}_{2,4}^{12,3}(36 n+15) q^{n} \equiv 2 f_{3} f_{6}(\bmod 4)
$$

Congruences (1.28) and (1.29) follow by extracting the terms involving $q^{3 n+1}$ and $q^{3 n+2}$ from (9.9).

From (9.12), we find that

$$
\sum_{n=0}^{\infty} \bar{B}_{2,4}^{12,3}(108 n+15) q^{n} \equiv 2 f_{1} f_{2}(\bmod 4) .
$$

Combining (9.10) and (9.13), we get

$$
\bar{B}_{2,4}^{12,3}(108 n+15) \equiv \bar{B}_{2,4}^{12,3}(12 n+3)(\bmod 4) .
$$

Using the aforementioned relation and by induction on $\alpha$, we have

$$
\bar{B}_{2,4}^{12,3}\left(4 \cdot 3^{2 \alpha+3} n+\frac{3 \cdot\left(3^{2 \alpha+2}+1\right)}{2}\right) \equiv \bar{B}_{2,4}^{12,3}(12 n+3)(\bmod 4) .
$$

Using (1.28) in (9.15), we obtain (1.30). 
10. Proof of Theorem (1.8)

28,2

From (9.11), we find that

$$
\sum_{n=0}^{\infty} \bar{B}_{2,4}^{12,3}(36 n+3) q^{n} \equiv 2 \frac{f_{2} f_{3}^{4}}{f_{1} f_{6}^{2}}(\bmod 4) .
$$

Invoking (2.11) in (10.1), we obtain

$$
\sum_{n=0}^{\infty} \bar{B}_{2,4}^{12,3}(36 n+3) q^{n} \equiv 2 f_{1}(\bmod 4) .
$$

Employing Lemma (2.7) into (10.2), it can be see that

$$
\sum_{n=0}^{\infty} \bar{B}_{2,4}^{12,3}\left(36\left(p n+\frac{p^{2}-1}{24}\right)+3\right) q^{n} \equiv 2 f_{p}(\bmod 4),
$$

which implies

$$
\sum_{n=0}^{\infty} \bar{B}_{2,4}^{12,3}\left(36 p^{2} n+\frac{3\left(p^{3}+1\right)}{2}\right) q^{n} \equiv 2 f_{1}(\bmod 4) .
$$

Therefore,

$$
\bar{B}_{2,4}^{12,3}\left(36 p^{2} n+\frac{3\left(p^{3}+1\right)}{2}\right) \equiv \bar{B}_{2,4}^{12,3}(36 n+3)(\bmod 4) .
$$

Using the aforementioned relation and by induction on $\alpha$, we arrive at (1.31).

\section{Proof of Theorem (1.9)}

Combining (10.3) with Theorem (1.8), we derive that for $\alpha \geq 0$,

$$
\sum_{n=0}^{\infty} \bar{B}_{2,4}^{12,3}\left(36 p^{2 \alpha+1} n+\frac{3\left(p^{3 \alpha}+1\right)}{2}\right) \equiv 2 f_{p}(\bmod 4) .
$$

Therefore, it follows that

$$
\sum_{n=0}^{\infty} \bar{B}_{2,4}^{12,3}\left(36 p^{2 \alpha+1}(p n+l)+\frac{3\left(p^{3 \alpha}+1\right)}{2}\right) \equiv 0(\bmod 4) .
$$

where $l=1,2, \ldots, p-1$, we obtain (1.32).

\section{Acknowledgement:}

The authors would like to thank the anonymous referee for his valuable suggestions to improve the quality of our paper.

Funding: This research received no specific grants from any funding agency in the public, commercial or not for profit sectors.

\section{References}

1. Berndt BC, Ramanujan's notebooks Part III, New York, NY: Springer-Verlag; 1991. 
2. Corteel S, Lovejoy J, Overpartitions, Trans Amer Math Soc. 2004; 356: 1623-35.

3. Andrews GE, Singular overpartitions, Int J Number Theory. 2015; 11: 1523-33.

4. Lovejoy J, Gordon's theorem for overpartitions, J Comb Thy A. 2003; 103: 393-401.

5. Chen SC, Hirschhorn MD, Sellers JA, Arithmetic properties of Andrews' singular overpartitions, Int J Number Theory. 2015; 11: 1463-76.

6. Ahmed Z, Baruah ND, New congruences for Andrews' singular overpartitions, Int. J Number Theory. 2015; 11: 2247-64.

7. Chen SC, Congruences and asymptotics of Andrews' singular overpartitions, J Number Theory. 2016; 164: 343-58.

8. Yao OXM, Congruences modulo 16, 32, and 64 for Andrews' singular overpartitions, Ramanujan J. 2017; 43: 215-28.

9. Mahadeva Naika MS, Gireesh DS, Congruences for Andrews' singular overpartitions, J. Number Theory. 2016; 165: 109-30.

10. Mahadeva Naika MS, Shivaprasada Nayaka S, Andrews' singular overpartitions with odd parts, Funct Approx Comment Math. 2017; 56(2): 195-209.

11. Mahadeva Naika MS, Shivaprasada Nayaka S, Andrews' singular overpartitions without multiples of $k$, Gulf J Math. 2017; 5(1): 18-32.

12. Mahadeva Naika MS, Shivashankar C, Congruences for Andrews' singular overpartition pairs, Int J Number Theory. 2018; 14(4): 989-1008.

13. Mahadeva Naika MS, Shivaprasada Nayaka S, Some new congruences for Andrews' singular overpartition pairs, Vietnam J Math. 2018; 46: 609-28.

14. Hirschhorn MD, Garvan F, Borwein J, Cubic analogs of the Jacobian cubic theta function $\theta(z, q)$, Canad J Math. 1993; 45: 673-94.

15. Baruah ND and Ojah KK, Analogues of Ramanujan's partition identities and congruences arising from the theta functions and modular equations, Ramanujan J. 2012; 28: 385-407.

16. Baruah ND, Ojah KK, Partitions with designated summands in which all parts are odd, Integers. 2015; 15(A9): 16.

17. Xia EXW, Yao OXM, New Ramanujan-like congruences modulo powers of 2 and 3 for overpartitions, J. Number Theory. 2013; 133: 1932-49.

18. Xia EXW, Yao OXM, Analogues of Ramanujan's partition identities, Ramanujan J. 2013; 31: 373-96.

19. Hirschhorn MD, Sellers JA, Elementary proofs of parity results for 5-regular partitions, Bull Aust Math Soc. 2010; 81: 58-63.

20. Hirschhorn MD, Sellers JA, A congruence modulo 3 for partitions into distinct non-multiples of four, J Integer Seq. 2014; 17: 14.9.6.

21. Cui SP, Gu NSS, Arithmetic properties of $\ell$-regular partitions, Adv Appl Math. 2013; 51: 507-23.

\section{Corresponding author}

S. Shivaprasada Nayaka can be contacted at: shivprasadnayaks@bmsit.in

For instructions on how to order reprints of this article, please visit our website:

www.emeraldgrouppublishing.com/licensing/reprints.htm

Or contact us for further details: permissions@emeraldinsight.com 